\title{
EDITORIAL
}

\section{Hypoglossal neurostimulation for obstructive sleep apnoea}

\author{
Ferran Barbé $^{\star, \#}$ and Juan F. Masa ${ }^{\#, \uparrow}$
}

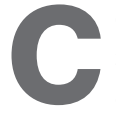

ontinuous positive airway pressure (CPAP) is the goldstandard treatment for patients with obstructive sleep apnoea (OSA). Since its introduction in 1981 [1], hundreds of thousands of patients have been treated successfully all over Europe. CPAP treatment improves clinical symptoms, reduces road accidents, decreases blood pressure and probably lowers cardiovascular risk [2]. All these positive effects of CPAP treatment are dependent, however, on compliance with the treatment. As in any other chronic disease, $\sim 30 \%$ of OSA patients refuse treatment or do not follow it as required. The alternative treatments currently available for OSA are surgery, oral appliances and weight reduction. The success rate of such treatments is low and they are mainly reserved for patients with mild-to-moderate OSA.

OSA is caused by an intermittent collapse of the upper airway. Since the seminal study by REMMERs et al. [3], it has been known that this obstruction is related to a loss in the patency of the genioglossus muscle, the main dilator of the upper airway. Although electrical stimulation of pharyngeal muscles during sleep demonstrated its efficacy in studies in the mid-1990s, it was never successfully developed for use in clinical practice [4, 5]. More recently, various research groups have shown increasing interest in this potential treatment, generally by implanting an electrode in the hypoglossal nerve to allow activation of the protrusor muscles of the tongue. This activation is intermittent and synchronic with the respiratory cycle, which is monitored with implanted thoracic leads that sense movement in the wall of the chest $[6,7]$. Other authors have performed a continuous submental electrical stimulation [8]. Generally speaking, the results have not been outstanding and the improvement in sleep respiratory disturbances has only been partial. Moreover, serious device-related adverse effects have been reported. In this issue of the European Respiratory Journal, MWENGE et al. [9] report their experience with continuous (nonsynchronic with the respiratory cycle) hypoglossal neurostimulation in OSA patients. They performed an open-label, single-site study to evaluate the safety and efficacy of hypoglossal neurostimulation for sleep apnoea. They included patients with an apnoea-hypopnoea index (AHI) $\geqslant 20$ events $\cdot h^{-1}$ who had refused CPAP treatment. There was no

\footnotetext{
*Respiratory Dept, Hospital Universitari Arnau de Vilanova-Santa María, IRB Lleida, Lleida, ${ }^{\#}$ Centro de Investigación Biomédica en Red de Enfermedades Respiratorias, Madrid, and "Respiratory Dept, Hospital San Pedro de Alcantara, Caceres, Spain.

CORRESPONDENCE: F. Barbé, Respiratory Dept, Hospital Universitari Arnau de Vilanova-Santa María, IRB Lleida, Rovira Roure 80, 25198 Lleida, Spain. E-mail: fbarbe@arnau.scs.es
}

preferential selection of subjects by apnoea or hypopnoea indices. 13 patients were evaluated after 12 months of treatment.

This study raises some intriguing questions. The first question is: does it work? The answer is yes, it does, but only partially. Continuous hypoglossal neurostimulation improved oxygen desaturation, as well as the arousal index and the Epworth Sleepiness Scale score. The AHI improved from $45 \pm 18$ to $21 \pm 17$ events $\cdot h^{-1}$. The reduction in the AHI was $>50 \%$, which is an acceptable result rate for some physicians, although an elevated residual AHI was still found in some subjects. Four of the 13 patients showed a residual AHI $>20$ events $\cdot h^{-1}$ and the treatment failed to obtain satisfactory clinical results in three of these cases. Overall, this study shows a higher rate of response than previous ones $[6,7]$. These relatively positive results should be evaluated after analysis of the presence of relevant secondary adverse effects. There were two patients with transient (months) tongue paresis, one patient with postoperative swelling that lasted 2 weeks, three cables broke in two patients and two patients had technical problems with the implanted pulse generator. What is interesting about this study, however, is that the authors did not exclude patients with severe OSA, and showed that hypoglossal neurostimulation improves objective data and symptoms in patients with severe OSA who refuse CPAP treatment.

The second question is: can this procedure be performed anywhere? Probably not yet. The implantation is performed under general anaesthesia and requires hypoglossal nerve dissection. The electrode is rolled around the main trunk of the nerve and the pulse generator is placed in a subcutaneous pectoral pocket. This procedure requires some expertise although, as MwENGE et al. [9] observe, surgeons well trained in ear, nose and throat, or maxillofacial surgery should be able to perform this implantation without any problem. The second specific procedure is neurostimulation titration. This requires titration during the day, while the patient remains awake, and at night, during a polysomnographic study; finally, the titration is adjusted in a second night study. This procedure is cumbersome and should be simplified and standardised.

The third question is related to cost. The procedure needs to be evaluated in overall financial terms. This treatment seems to be very expensive when compared with other treatments such as CPAP. In addition to the surgical procedure, which requires 1 day of hospitalisation, there is the cost of the sleep studies needed to titrate the procedure (at least two) and that of the close follow-up required. It is also necessary to add the costs of the electrode and the pulse generator, which must be changed 
periodically. In future, the surgical procedure, which does not seem to be particularly difficult, may become a technique appropriate for outpatients. Furthermore, the titration procedure should be simplified and it may be possible that titration is only needed during the day. These improvements in the treatment procedure will decrease the cost of the treatment.

The last question is: how does it work? We do not know. The mechanism that explains the effect of neurostimulation in nerves or muscles is unknown. One could speculate that such stimulation trains muscles and improves their endurance, but this hypothesis needs to be proven. In their study, MWENGE et al. [9] used continuous (nonsynchronic with the respiratory cycle) but cyclical stimulation of the hypoglossus to ensure that no single nerve fibre was continuously stimulated. This should minimise the risk of nerve or muscle fatigue. The authors sought to stimulate an undetermined number of tongue muscles, both protractor and retractor, in order to achieve favourable net movement in both the pharynx and mouth (a concept consistent with the hydrostat model of the tongue).

In future, it will be necessary to select patients adequately and exclude nonresponders (e.g. patients with severe obesity) a priori. Craniofacial structures and body position could also condition the response to hypoglossal neurostimulation. It will also be very exciting to explore the effects of bilateral hypoglossal neurostimulation. Bilateral stimulation may be more effective and could be performed in the most difficult cases. Technical evolution can also be expected to improve patients' acceptance of the treatment: it will not be necessary to recharge the battery generator for an hour every day; and it will be easy to monitor compliance, in technical terms. There is still a long way to go before hypoglossal nerve stimulation becomes a real alternative to CPAP treatment, but the study by MWENGE et al. [9] is the most relevant to date and points in that direction.

\section{STATEMENT OF INTEREST}

None declared.

\section{REFERENCES}

1 Sullivan CE, Issa FG, Berthon-Jones M, et al. Reversal of obstructive sleep apnoea by continuous positive airway pressure applied through the nares. Lancet 1981; 1: 862-865.

2 Barbé F, Durán-Cantolla J, Sánchez-de-la-Torre M, et al. Effect of continuous positive airway pressure on the incidence of hypertension and cardiovascular events in nonsleepy patients with obstructive sleep apnea: a randomized controlled trial. JAMA 2012; 307: 2161-2168.

3 Remmers JE, deGroot WJ, Sauerland EK, et al. Pathogenesis of upper airway occlusion during sleep. J Appl Physiol 1978; 44: 931-938.

4 Eisele DW, Smith PL, Alam DS, et al. Direct hypoglossal nerve stimulation in obstructive sleep apnea. Arch Otolaryngol Head Neck Surg 1997; 123: 57-61.

5 Schwartz AR, Bennett ML, Smith PL, et al. Therapeutic electrical stimulation of the hypoglossal nerve in obstructive sleep apnea. Arch Otolaryngol Head Neck Surg 2001; 127: 1216-1223.

6 Van de Heyning PH, Badr MS, Baskin JZ, et al. Implanted upper airway stimulation device for obstructive sleep apnea. Laryngoscope 2012; 122: 1626-1633.

7 Eastwood PR, Barnes M, Walsh JH, et al. Treating obstructive sleep apnea with hypoglossal nerve stimulation. Sleep 2011; 34: 1479-1486.

8 Steier J, Seymour J, Rafferty GF, et al. Continuous transcutaneous submental electrical stimulation in obstructive sleep apnea: a feasibility study. Chest 2011; 140: 998-1007.

9 Mwenge GB, Rombaux P, Dury M, et al. Targeted hypoglossal neurostimulation for obstructive sleep apnoea: a 1-year pilot study. Eur Respir J 2013; 41: 360-367. 